International Journal of Pure and Applied Mathematics

Volume 85 No. 3 2013, 531-539

ISSN: 1311-8080 (printed version); ISSN: 1314-3395 (on-line version)

url: http://www.ijpam.eu

doi: http://dx.doi.org/10.12732/ijpam.v85i3.8

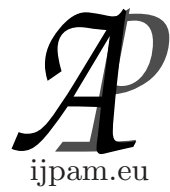

\title{
THE LAPLACIAN ON AN AFFINE HOMOGENEOUS SPACE
}

\author{
Liangzhong $\mathrm{Hu}^{1}$, José João Rossetto ${ }^{2}$ \\ 1,2Department of Mathematics \\ Federal University of Paraná \\ C.P. 019081, Curitiba, PR, 81531-990, BRAZIL
}

\begin{abstract}
The solution of the eigenvalue problem of the Laplacian on the affine homogeneous space $\hat{\mathrm{g}} / \hat{\boldsymbol{\eta}}$ is obtained. Here $\hat{\mathrm{g}}$ and $\hat{\boldsymbol{\eta}}$ are affine Lie algebras, $\hat{\mathrm{g}}=\mathbf{g} \otimes \mathbf{C}\left[t, t^{-1}\right] \oplus \mathbf{C} \hat{k} \oplus \mathbf{C} d$ and $\hat{\boldsymbol{\eta}}=\boldsymbol{\eta} \otimes \mathbf{C}\left[t, t^{-1}\right] \oplus \mathbf{C} \hat{k} \oplus \mathbf{C} d . \boldsymbol{\eta} \subset \mathbf{g}$ are two Lie algebras with $\mathbf{g}$ semi-simple and $\boldsymbol{\eta}$ reductive and having the same rank.
\end{abstract}

AMS Subject Classification: 22E65, 51P05, 81R10

Key Words: Laplacian, affine homogeneous space, Weyl group, highest weight

\section{Introduction}

Homogeneous spaces play an important role in mathematics and physics, (see, for example [1], [2], [3], [4] and references therein).

In [5], we gave the solution of the eigenvalue problem of the Laplacian on a homogeneous space $G / H$, where $G$ is a compact, semi-simple Lie group, $H$ is a closed subgroup of $G$, and the rank of $H$ is equal to the rank of $G$.

In this paper we generalize the result and give the solution of the eigenvalue problem of the Laplacian on $\hat{\mathbf{g}} / \hat{\boldsymbol{\eta}}$. Here $\hat{\mathrm{g}}$ and $\hat{\boldsymbol{\eta}}$ are affine Lie algebras, $\hat{\mathrm{g}}=$ $\mathbf{g} \otimes \mathbf{C}\left[t, t^{-1}\right] \oplus \mathbf{C} \hat{k} \oplus \mathbf{C} d$ and $\hat{\boldsymbol{\eta}}=\boldsymbol{\eta} \otimes \mathbf{C}\left[t, t^{-1}\right] \oplus \mathbf{C} \hat{k} \oplus \mathbf{C} d \cdot \boldsymbol{\eta} \subset \mathbf{g}$ are two Lie algebras with $\mathbf{g}$ semi-simple and $\boldsymbol{\eta}$ reductive and having the same rank.

Received: January 30, 2013

(C) 2013 Academic Publications, Ltd.

$\S$ Correspondence author url: www.acadpubl.eu 
The layout of the paper is as follows. In Section 2, we review the result in [5]. In Secction 3, we first give the definition of the Laplacian on $\hat{\mathbf{g}} / \hat{\boldsymbol{\eta}}$. Then we give the eigenvalues of the Laplacian on $\hat{\mathbf{g}} / \hat{\boldsymbol{\eta}}$. Finally, The lowest eigenvalue and its eigenspace of the Laplacian on $\hat{\mathbf{g}} / \hat{\boldsymbol{\eta}}$ is obtained.

\section{The Laplacian on $G / H$}

We briefly review the solution of the eigenvalue problem of the Laplacian on homogeneous space $G / H$. Here $G$ is a compact, semi-simple Lie group, $H$ is a closed subgroup of $G$, and the rank of $H$ is equal to the rank of $G$. More detailed account can be found in [5].

Let $\mathbf{g}$ and $\boldsymbol{\eta}$ be the Lie algebras of $G$ and $H$, respectively.

We suppose that $G / H$ is reductive, i.e. g has an orthognal decomposition $\mathbf{g}=\boldsymbol{\eta} \oplus \mathbf{m}$ with $[\boldsymbol{\eta}, \mathbf{m}] \subset \mathbf{m}$ and $[\mathbf{m}, \mathbf{m}] \subset \mathbf{g}$.

We can choose a common Cartan subalgebra

$$
\mathbf{h} \subset \boldsymbol{\eta} \subset \mathbf{g} .
$$

Let $\Phi_{\mathrm{g}}$ be the set of roots of $\mathrm{g}$. The roots $\Phi_{\boldsymbol{\eta}}$ of $\boldsymbol{\eta}$ form a subset of the roots of $\mathrm{g}$, i.e.,

$$
\Phi_{\eta} \subset \Phi_{\mathrm{g}}
$$

Choosing a positive root system $\Phi_{\mathrm{g}}^{+}$for $\mathbf{g}$ also determines a positive root system $\Phi_{\boldsymbol{\eta}}^{+}$for $\boldsymbol{\eta}$, where

$$
\Phi_{\eta}^{+} \subset \Phi_{\mathrm{g}}^{+}
$$

Let $\rho_{\mathbf{g}}=\frac{1}{2} \sum_{\alpha \in \Phi_{\mathrm{g}}^{+}} \alpha$ and $\rho_{\boldsymbol{\eta}}=\frac{1}{2} \sum_{\alpha \in \Phi_{\boldsymbol{\eta}}^{+}} \alpha$ denote the Weyl vector of $\mathbf{g}$ and $\boldsymbol{\eta}$ respectively.

Let $U_{\mu}$ be a given irreducible representation of $\boldsymbol{\eta}$ with highest weight $\mu$. Let $G \times{ }_{H} U$ be the associated vector bundle of the principal bundle $P(G / H, H)$. The Hilbert space of square integrable sections of $G \times{ }_{H} U_{\mu}$ decomposes into the direct sum of the eigenspaces of the Laplacian on $G / H$, which are irreducible representations $V_{\lambda}$ of $\mathbf{g}$ with highest weights $\lambda$ 's. and this induces the following expression for the Laplacian on $G / H$ which was discussed in [6], [7], [8], [9], [10], [11], [12], [13], [14], [15], [16], [17], [18] and appears explicitly in [19].

Definition 1. The Laplacian on $G / H$ is

$$
\Delta=C_{2}(\mathbf{g}, \cdot)-C_{2}(\boldsymbol{\eta}, U) .
$$

Here $C_{2}(\mathbf{g}, \cdot)$ is the quadratic Casimir element of $\mathbf{g}$ calculated in an irreducible representation of $\mathbf{g} . C_{2}(\boldsymbol{\eta}, U)$ is the quadratic Casimir element of $\boldsymbol{\eta}$ calculated in a given irreducible representation $U$. 
Thus we have the following result.

Theorem 2. Given an irreducible representation $U_{\mu}$ of $\boldsymbol{\eta}$ with highest weight $\mu$. The eigenvalue of $\Delta$ labelled by a highest weight $\lambda$ reads

$$
E_{\lambda}=\left(\lambda+\rho_{\mathbf{g}}, \lambda+\rho_{\mathbf{g}}\right)-\left(\mu+\rho_{\boldsymbol{\eta}}, \mu+\rho_{\boldsymbol{\eta}}\right)-\left(\rho_{\mathbf{g}}, \rho_{\mathbf{g}}\right)+\left(\rho_{\boldsymbol{\eta}}, \rho_{\boldsymbol{\eta}}\right)
$$

with

$$
\left(\lambda+\rho_{\mathbf{g}}, \lambda+\rho_{\mathbf{g}}\right) \geq\left(\mu+\rho_{\boldsymbol{\eta}}, \mu+\rho_{\boldsymbol{\eta}}\right) .
$$

The multiplicity of the eigenvalue $E_{\lambda}$ is given by the Weyl dimension formula:

$$
\operatorname{dim} V_{\lambda}=\frac{\prod_{\alpha \in \Phi_{\mathbf{g}}^{+}}\left(\lambda+\rho_{\mathbf{g}}, \alpha\right)}{\prod_{\alpha \in \Phi_{\mathbf{g}}^{+}}\left(\rho_{\mathbf{g}}, \alpha\right)} .
$$

Moreover, If there exists an element $w \in W_{\mathbf{g}}$ in the Weyl group of $\mathbf{g}$ such that the weight $w\left(\mu+\rho_{\boldsymbol{\eta}}\right)-\rho_{\mathbf{g}}$ is dominant for $\mathbf{g}$. Then the lowest eigenvalue of $\Delta$ is

$$
E_{w\left(\mu+\rho_{\boldsymbol{\eta}}\right)-\rho_{\mathbf{g}}}=\left(\rho_{\boldsymbol{\eta}}, \rho_{\boldsymbol{\eta}}\right)-\left(\rho_{\mathbf{g}}, \rho_{\mathbf{g}}\right),
$$

and the multiplicity of the lowest eigenvalue of $\Delta$ is

$$
\operatorname{dim} V_{w\left(\mu+\rho_{\boldsymbol{\eta}}\right)-\rho_{\mathbf{g}}}=\frac{\prod_{\alpha \in \Phi_{\mathbf{g}}^{+}}\left(w\left(\mu+\rho_{\boldsymbol{\eta}}\right), \alpha\right)}{\prod_{\alpha \in \Phi_{\mathbf{g}}^{+}}\left(\rho_{\mathbf{g}}, \alpha\right)} .
$$

Remark. 1. If $\lambda=w\left(\mu+\rho_{\boldsymbol{\eta}}\right)-\rho_{\mathbf{g}}$ is not dominant for $\mathbf{g}$, the lowest eigenvalue of $\Delta$ does not exist. Thus we can always choose $\mu$ such that $\lambda$ is dominant.

2. $V_{w\left(\mu+\rho_{\eta}\right)-\rho_{\mathrm{g}}}$ is, up to a sign, equal to the $G$-equivariant index of the Kostant's Dirac operator on $G / H$ [20], [21], [22], [23].

\section{The Solution of Eigenvalue Problem of the Laplacian on $\hat{g} / \hat{\eta}$}

\subsection{The Laplacian and its Eigenvalues on $\hat{\mathrm{g}} / \hat{\eta}$}

An introduction to the affine Lie algebra can be found in [24], [4]. Let $\hat{\mathrm{g}}=$ $\mathbf{g} \otimes \mathbf{C}\left[t, t^{-1}\right] \oplus \mathbf{C} \hat{k} \oplus \mathbf{C} d$ be an affine Lie algebra. Here $\mathbf{C}\left[t, t^{-1}\right]$ is the set of Laurent polinomials in the variable $t, \hat{k}$ is the central extension operator and $d$ is the energy operator. Let $\hat{\lambda}=\left(\lambda ; k_{\lambda} ; n_{\lambda}\right)$ and $\hat{\mu}=\left(\mu ; k_{\mu} ; n_{\mu}\right)$ be two affine weights of $\hat{\mathbf{g}}$. Here $\lambda$ and $\mu$ are weights of $\mathbf{g}, k_{\lambda}$ and $k_{\mu}$ are levels, $n_{\lambda}$ and $n_{\mu}$ 
are energies. The scalar product of $\hat{\lambda}$ and $\hat{\mu}$ induced by the extended Killing form is

$$
(\hat{\lambda}, \hat{\mu})=(\lambda, \mu)+k_{\lambda} n_{\mu}+k_{\mu} n_{\lambda} .
$$

The Casimir operator $C_{2}(\hat{\mathbf{g}})$ on $\hat{\mathbf{g}}$ is the generalization of the Casimir operator on $\mathbf{g}$. The detailed account for the construction can be found, for example, in $[25],[26]$.

$$
\begin{aligned}
C_{2}(\hat{\mathbf{g}})= & \sum_{a=1}^{l} \sum_{m \in \mathbf{Z}}: X_{m}^{a} X_{-m}^{a}:+2(k+g) d \\
= & \sum_{a=1}^{l}\left[\sum_{m \leq-1} X_{m}^{a} X_{-m}^{a}+\sum_{m \geq 0} X_{-m}^{a} X_{m}^{a}\right] \\
& +2(k+g) d .
\end{aligned}
$$

Here : : is the usual normal ordering, $g$ is the dual Coxeter number of $\mathbf{g}, X_{m}^{a}=$ $X^{a} \otimes t^{m}$ with $X^{a}$ generators of $\mathbf{g}, l$ is the dimension of $\mathbf{g}$ and $\mathbf{Z}$ is the set of integers. The Casimir operator commutes with $\hat{\mathbf{g}}$. If $V_{\hat{\lambda}}$ is a highest weight representation generated by a highest weight vector $\mid \hat{\lambda}>$ of $\hat{\lambda}=(\lambda ; k ; n)$, then

$$
\begin{aligned}
C_{2}(\hat{\mathbf{g}}) \mid \hat{\lambda}>= & {\left[\sum_{a=1}^{l} X_{0}^{a} X_{0}^{a}+2(k+g) d\right] \mid \hat{\lambda}>} \\
= & {\left[\sum_{a=1}^{l} X^{a} X^{a}+2(k+g) d\right] \mid \hat{\lambda}>} \\
= & {\left[\left(\hat{\lambda}+\hat{\rho}_{\hat{\mathbf{g}}}, \hat{\lambda}+\hat{\rho}_{\hat{\mathbf{g}}}\right)-\left(\hat{\rho}_{\hat{\mathbf{g}}}, \hat{\rho}_{\hat{\mathbf{g}}}\right)\right] \mid \hat{\lambda}>} \\
= & {\left[\left(\lambda+\rho_{\mathbf{g}}, \lambda+\rho_{\mathbf{g}}\right)-\left(\rho_{\mathbf{g}}, \rho_{\mathbf{g}}\right)\right.} \\
& +2(k+g) n] \mid \hat{\lambda}>,
\end{aligned}
$$

where $\hat{\rho}_{\hat{\mathbf{g}}}=\left(\rho_{\mathbf{g}} ; g ; 0\right)$ is the affine Weyl vector of $\hat{\mathrm{g}}$ with $g$ the dual Coxeter number of $\mathbf{g}$. The following convention is often adopted (see [4]).

Convention 3. For a highest weight, $\hat{\lambda}(d)=n=0$.

One has

$$
C_{2}(\hat{\mathbf{g}})\left|\hat{\lambda}>=\left[\left(\lambda+\rho_{\mathbf{g}}, \lambda+\rho_{\mathbf{g}}\right)-\left(\rho_{\mathbf{g}}, \rho_{\mathbf{g}}\right)\right]\right| \hat{\lambda}>=C_{2}(\mathbf{g}) \mid \hat{\lambda}>.
$$

The Laplacian on $\hat{\mathbf{g}} / \hat{\boldsymbol{\eta}}$ is the generalization of the Laplacian on $\mathbf{g} / \boldsymbol{\eta}$. We have

Definition 4. The Laplacian on $\hat{\mathrm{g}} / \hat{\boldsymbol{\eta}}$ is

$$
\hat{\Delta}=C_{2}(\hat{\mathbf{g}}, \cdot)-C_{2}(\hat{\boldsymbol{\eta}}, U) .
$$

Here $C_{2}(\hat{\mathbf{g}}, \cdot)$ is the Casimir operator of $\hat{\mathbf{g}}$ calculated in an irreducible representation of $\hat{\mathbf{g}}$. $C_{2}(\hat{\boldsymbol{\eta}}, U)$ is the Casimir operator of $\hat{\boldsymbol{\eta}}$ calculated in a given irreducible representation $U$. 
Proposition 5. Given an irreducible representation $U_{\hat{\mu}}$ of $\hat{\boldsymbol{\eta}}$ with highest weight $\hat{\mu}=\left(\mu ; k_{\mu} ; 0\right)$. The eigenvalue of $\hat{\Delta}$ on $\hat{\mathbf{g}} / \hat{\boldsymbol{\eta}}$ labelled by highest weight $\hat{\lambda}=\left(\lambda ; k_{\lambda} ; 0\right)$ reads

$$
\begin{aligned}
E_{\hat{\lambda}}= & \left(\hat{\lambda}+\hat{\rho}_{\hat{\mathbf{g}}}, \hat{\lambda}+\hat{\rho}_{\hat{\mathbf{g}}}\right)-\left(\hat{\mu}+\hat{\rho}_{\hat{\boldsymbol{\eta}}}, \hat{\mu}+\hat{\rho}_{\hat{\boldsymbol{\eta}}}\right)-\left(\hat{\rho}_{\hat{\mathbf{g}}}, \hat{\rho}_{\hat{\mathbf{g}}}\right)+\left(\hat{\rho}_{\hat{\boldsymbol{\eta}}}, \hat{\rho}_{\hat{\boldsymbol{\eta}}}\right) \\
= & \left(\lambda+\rho_{\mathbf{g}}, \lambda+\rho_{\mathbf{g}}\right)-\left(\mu+\rho_{\boldsymbol{\eta}}, \mu+\rho_{\boldsymbol{\eta}}\right) \\
& -\left(\rho_{\mathbf{g}}, \rho_{\mathbf{g}}\right)+\left(\rho_{\boldsymbol{\eta}}, \rho_{\boldsymbol{\eta}}\right)
\end{aligned}
$$

with

$$
\left(\lambda+\rho_{\mathbf{g}}, \lambda+\rho_{\mathbf{g}}\right) \geq\left(\mu+\rho_{\boldsymbol{\eta}}, \mu+\rho_{\boldsymbol{\eta}}\right) .
$$

The corresponding eigenspace is the irreducible representation of $\hat{\mathbf{g}}, V_{\left(\lambda ; k_{\lambda} ; 0\right)}$ with highest weight $\left(\lambda ; k_{\lambda} ; 0\right)$.

\subsection{The Lowest Eigenvalue and its Eigenspace of $\hat{\Delta}$ on $\hat{g} / \hat{\eta}$}

In order to determine the lowest eigenvalue and its eigenspace of $\hat{\Delta}$ on $\hat{\mathrm{g}} / \hat{\boldsymbol{\eta}}$, we first review the relation between the Weyl groups $W_{\mathbf{g}}$ of $\mathbf{g}$ and $W_{\boldsymbol{\eta}}$ of $\boldsymbol{\eta}$ [21]. $W_{\boldsymbol{\eta}}$ is a subgroup of $W_{\mathbf{g}}$. Choose the positive roots consistently. Then the positive Weyl chamber $\mathcal{W}_{\mathbf{g}}$ of $\mathbf{g}$ is contained in the positive Weyl chamber $\mathcal{W}_{\boldsymbol{\eta}}$ of $\boldsymbol{\eta}$. Let

$$
C \subset W_{\mathrm{g}}
$$

denote the set of elements that map $\mathcal{W}_{\mathbf{g}}$ into $\mathcal{W}_{\boldsymbol{\eta}}$. So the cardinality of $C$ is the index of $W_{\boldsymbol{\eta}}$ in $W_{\mathbf{g}}$, and

$$
\mathcal{W}_{\boldsymbol{\eta}}=\bigcup_{c \in C} c\left(\mathcal{W}_{\mathbf{g}}\right)
$$

while

$$
W_{\mathrm{g}}=W_{\boldsymbol{\eta}} \cdot C .
$$

Let $\lambda$ be a dominant weight of $\mathbf{g}$ and $V_{\lambda}$ be the corresponding irreducible representation of $\mathbf{g}$. For each $c \in C$, let

$$
c \bullet \lambda=c\left(\lambda+\rho_{\mathbf{g}}\right)-\rho_{\boldsymbol{\eta}}
$$

Then $c \bullet \lambda$ is a dominant weight for $\boldsymbol{\eta}$.

It can be seen that the above relations are also satisfied in the corresponding affine Weyl groups. Now we generalize the result in Section 2 to the case of the affine Lie algebras. Given an irreducible representation $U_{\hat{\mu}}$ of $\hat{\boldsymbol{\eta}}$ with highest weight $\hat{\mu}, \hat{\mu}$ corresponds to a unique highest weight $\hat{\lambda}$ of an irreducible 
representation $V_{\hat{\lambda}}$ of $\hat{\mathbf{g}}$. More precisely, there exists $c \in \hat{C} \subset \hat{W}_{\hat{\mathbf{g}}}$, where $\hat{W}_{\hat{\mathbf{g}}}$ is the affine Weyl group of $\hat{\mathbf{g}}$, such that $\hat{\mu}=c \bullet \hat{\lambda}=c\left(\hat{\lambda}+\hat{\rho}_{\hat{\mathbf{g}}}\right)-\hat{\rho}_{\hat{\boldsymbol{\eta}}}$, where $\hat{\rho}_{\hat{\mathbf{g}}}=\left(\rho_{\mathbf{g}} ; g ; 0\right)$ and $\hat{\rho}_{\hat{\boldsymbol{\eta}}}=\left(\rho_{\boldsymbol{\eta}} ; \eta ; 0\right)$ are affine Weyl vectors of $\hat{\mathbf{g}}$ and $\hat{\boldsymbol{\eta}}$, respectively; $g$ and $\eta$ are dual Coxeter numbers of $\mathbf{g}$ and $\boldsymbol{\eta}$, respectively. This means that

$$
\hat{\lambda}=w\left(\hat{\mu}+\hat{\rho}_{\hat{\boldsymbol{\eta}}}\right)-\hat{\rho}_{\hat{\mathbf{g}}}
$$

where $w=c^{-1} \in \hat{W}_{\hat{\mathbf{g}}}$. If $\hat{\lambda}$ is dominant for $\hat{\mathbf{g}}$, the eigenspace of the lowest eigenvalue is $V_{w\left(\hat{\mu}+\hat{\rho}_{\hat{\boldsymbol{\eta}}}\right)-\hat{\rho}_{\mathrm{g}}}$ with highest weight $w\left(\hat{\mu}+\hat{\rho}_{\hat{\boldsymbol{\eta}}}\right)-\hat{\rho}_{\hat{\mathrm{g}}}$.

Let $\hat{\lambda}=(\lambda ; k ; n)$ be a weight of $\hat{\mathbf{g}}(\hat{\boldsymbol{\eta}})$. Let $\hat{\alpha}=(\alpha ; 0 ; m)$ be an affine root of $\hat{\mathrm{g}}(\hat{\boldsymbol{\eta}})$. The Weyl reflection with respect to $\hat{\alpha}$ reads

$$
\begin{aligned}
s_{\hat{\alpha}} \hat{\lambda} & =\hat{\lambda}-\left(\hat{\lambda}, \hat{\alpha}^{\vee}\right) \hat{\alpha} \\
& =\left(\lambda-[(\lambda, \alpha)+k m] \alpha^{\vee} ; k ; n-[(\lambda, \alpha)+k m] \frac{2 m}{|\alpha|^{2}}\right) \\
& =\left(s_{\alpha}\left(\lambda+k m \alpha^{\vee}\right) ; k ; n-[(\lambda, \alpha)+k m] \frac{2 m}{|\alpha|^{2}}\right) .
\end{aligned}
$$

Here $\hat{\alpha}^{\vee}$ is affine coroot of $\hat{\alpha}$ and $\alpha^{\vee}$ is coroot of $\alpha$. Now we impose the condition that the value of $\hat{\lambda}(d)$ is unchanged under the Weyl reflection, This forces $m=0$, i. e.,

$$
\hat{\alpha}=(\alpha ; 0 ; 0)=\alpha,
$$

and (17) reduces to

$$
w \hat{\lambda}=(w \lambda ; k ; n)
$$

where $w \in W_{\mathbf{g}}$, the Weyl group of $\mathbf{g}$. It means that only the Weyl group does not change $\hat{\lambda}(d)$ and one can obtain the related affine weights just by making use of the Weyl group. For a highest affine weight $\hat{\lambda}$, We have the convention that $\hat{\lambda}(d)=0$. This greatly simplifies the calculation.

Given an irreducible representation $U_{\hat{\mu}}$ of $\hat{\boldsymbol{\eta}}$ with highest weight $\hat{\mu}=\left(\mu ; k_{\mu} ; 0\right)$, from (16) and (17),

$$
\hat{\lambda}=w\left(\hat{\mu}+\hat{\rho}_{\hat{\boldsymbol{\eta}}}\right)-\hat{\rho}_{\hat{\mathbf{g}}}=\left(w\left(\mu+\rho_{\boldsymbol{\eta}}\right)-\rho_{\mathbf{g}} ; k+\eta-g ; 0\right),
$$

where $w \in W_{\mathbf{g}}, g$ and $\eta$ are dual Coxeter numbers of $\mathbf{g}$ and $\boldsymbol{\eta}$, respectively. If $\hat{\lambda}$ is dominant for $\hat{\mathbf{g}}$, the eigenspace of the lowest eigenvalue is the irreducible representation of $\hat{\mathbf{g}}, V_{\left(w\left(\mu+\rho_{\boldsymbol{\eta}}\right)-\rho_{\mathbf{g}} ; k+\eta-g ; 0\right)}$ with highest weight $\left(w\left(\mu+\rho_{\boldsymbol{\eta}}\right)-\right.$ $\left.\rho_{\mathbf{g}} ; k+\eta-g ; 0\right)$. It follows that $\left(\hat{\lambda}+\hat{\rho}_{\hat{\mathbf{g}}}, \hat{\lambda}+\hat{\rho}_{\hat{\mathbf{g}}}\right)=\left(w\left(\mu+\rho_{\boldsymbol{\eta}}\right), w\left(\mu+\rho_{\boldsymbol{\eta}}\right)\right)=$ $\left(\mu+\rho_{\boldsymbol{\eta}}, \mu+\rho_{\boldsymbol{\eta}}\right)=\left(\hat{\mu}+\hat{\rho}_{\hat{\boldsymbol{\eta}}}, \hat{\mu}+\hat{\rho}_{\hat{\boldsymbol{\eta}}}\right)$. By Proposition 4 , the lowest eigenvalue is $E_{\hat{w}\left(\hat{\mu}+\hat{\rho}_{\hat{\boldsymbol{\eta}}}\right)-\hat{\rho}_{\boldsymbol{g}}}=\left(\rho_{\boldsymbol{\eta}}, \rho_{\boldsymbol{\eta}}\right)-\left(\rho_{\mathbf{g}}, \rho_{\mathbf{g}}\right)$. Thus we have the following result: 
Theorem 6. Given an irreducible representation $U_{\hat{\mu}}$ of $\hat{\boldsymbol{\eta}}$ with highest weight $\hat{\mu}=\left(\mu ; k_{\mu} ; 0\right)$. If there exists an element $w \in W_{\mathbf{g}}$ in the Weyl group of $\mathbf{g}$ such that the weight $w\left(\hat{\mu}+\hat{\rho}_{\hat{\boldsymbol{\eta}}}\right)-\hat{\rho}_{\hat{\mathrm{g}}}=\left(w\left(\mu+\rho_{\boldsymbol{\eta}}\right)-\rho_{\mathbf{g}} ; k+\eta-g ; 0\right)$ is dominant for $\hat{\mathrm{g}}$. Then the eigenspace of the lowest eigenvalue of $\hat{\Delta}$ is the irreducible representation of $\hat{\mathbf{g}}, V_{\left(w\left(\mu+\rho_{\boldsymbol{\eta}}\right)-\rho_{\mathbf{g}} ; k+\eta-g ; 0\right)}$ with highest weight $\left(w\left(\mu+\rho_{\boldsymbol{\eta}}\right)-\right.$ $\left.\rho_{\mathbf{g}} ; k+\eta-g ; 0\right)$. The lowest eigenvalue of $\hat{\Delta}$ is

$$
E_{w\left(\hat{\mu}+\hat{\rho}_{\hat{\eta}}\right)-\hat{\rho}_{g}}=\left(\rho_{\boldsymbol{\eta}}, \rho_{\boldsymbol{\eta}}\right)-\left(\rho_{\mathbf{g}}, \rho_{\mathbf{g}}\right) .
$$

Remark. Due to the convention $\hat{\lambda}(d)=0$ for highest weights, The eigenvalues of $\hat{\Delta}$ on $\hat{g} / \hat{\boldsymbol{\eta}}$ are the same as the eigenvalues of $\Delta$ on $\mathbf{g} / \boldsymbol{\eta}$. However, the corresponding eigenspaces are different.

If $\hat{\lambda}=\left(w\left(\mu+\rho_{\boldsymbol{\eta}}\right)-\rho_{\mathbf{g}} ; k+\eta-g ; 0\right)$ is not dominant for $\hat{\mathbf{g}}$, the lowest eigenvalue of $\hat{\Delta}$ does not exist. Thus we can always choose $\hat{\mu}$ such that $\hat{\lambda}$ is dominant. It can be shown that $\hat{\lambda}$ is dominant for $\hat{\mathrm{g}}$, if $w\left(\mu+\rho_{\boldsymbol{\eta}}\right)-\rho_{\mathbf{g}}$ is dominant for $\mathbf{g}$, and the level $k+\eta-g \geq\left(w\left(\mu+\rho_{\boldsymbol{\eta}}\right)-\rho_{\mathbf{g}}, \theta\right)$, where $\theta$ is the highest root of $\mathbf{g}$.

\section{References}

[1] S. Kobayashi and K. Nomizu, Foundations of Differential Geometry, Vol. II, Interscience, (1969).

[2] S. Helgason, Differential Geometry, Lie Groups and Symmetric Spaces, Academic Press, (1978).

[3] F. W. Warner, Foundations of Differentiable Manifolds and Lie Groups, Graduate Texts in Mathematics, Vol. 94, Springer-Verlag, New York, (1983).

[4] P. Di Francesco, P. Mathieu and D. Sénéchal, Conformal Field Theory, Springer-Verlag New York, Inc. (1997).

[5] L. Hu, The Laplacian on homogeneous spaces, J. Math. Phys., 49 (2008), 053513.

[6] C. N. Yang, Generalization of Diracs monopole to $S U_{2}$ gauge fields, $J$. Math. Phys., 19 (1978), 320-328.

[7] H. Muto and H. Urakawa, On the least positive eigenvalue of Laplacian for compact homogeneous spaces, Osaka J. Math., 21 (1980), 471-484 . 
[8] A. Salam and Strathdee, On Kaluza-Klein theory, Ann. Phys., 141 (1982), 316-352.

[9] M. Stone, Supersymmetry and the quantum mechanics of spin, Nucl. Phys. $B, 314$ (1989), 557-586.

[10] V. D. Lyakhovsky, N. N. Shtykov and D. V. Vassilevich, DeWitt-Schwinger expansion for projective and Grassmann spaces, Lett. Math. Phys., 21 (1991), 89-95.

[11] T. Jaroszewicz and P. S. Kurzepa, Polyakov spin and Laplacians on homogeneous spaces, Annals of Physics, 213 (1992), 135-165.

[12] N. P. Landsman, Induced representations, gauge fields, and quantization on homogeneous spaces, Rev. Math. Phys., 4 (1992), 503-528.

[13] P. Lévay, D. McMullan and I.Tsutsui, The canonical connection in quantum mechanics, J. Math. Phys., 37 (1996), 625-636.

[14] S.-C. Zhang and J. Hu, A four dimensional generalization of the quantum Hall effect, Science, 294 (2001), 823-828.

[15] D. Karabali and V. P. Nair, Quantum Hall effect in higher dimensions, Nucl. Phys. B, 641 (2002), 533-546.

[16] B. Dolan, The spectrum of the Dirac operator on coset spaces with homogeneous gauge fields, JHEP, 0305 (2003), 018.

[17] B. A. Bernevig, J. Hu, N. Toumbas and S.-C. Zhang, The eight dimensional quantum Hall effect and the octonions, Phys. Rev. Lett. 91 (2003), 236803236807.

[18] S. Bellucci, P. Y. Casteill and A. Nersessian, Four-dimensional Hall mechanics as a particle on CP(3), Phys. Lett. B, 574 (2003), 121-128.

[19] G. Meng, Geometric construction of the Quantum Hall Effect in all even dimensions, J. Phys. A, 369415 (2003), 9415-9424.

[20] R. Bott, The index theorem for homogeneous differential operators, In: Differential and Combinatorial Topology, edited by S. S. Cairns, Princeton University Press, (1965), 167-186.

[21] B. Gross, B. Kostant, P. Ramond and S. Sternberg, The Weyl character formula, the half-spin representations, and equal rank subgroups, Proc. Natl. Acad. Sci. USA, 95 (1998), 8441-8442. 
[22] B. Kostant, A cubic Dirac operator and the emergence of the Euler number multiplets of representations for equal rank subgroups, Duke Math. J., 100 (1999), 447-501.

[23] G. D. Landweber, Harmonic spinors on homogeneous spaces, Representation Theory, 4 (2000), 466-473.

[24] V. G. Kac, Infinite Dimensional Lie Algebras, Third edition, Cambridge University Press, Cambridge (1990).

[25] A. Pressley and G. Segal, Loop Groups, Oxford University Press, (1988).

[26] A. Wassermann, Kac-Moody and Virasoro Algebras, arXiv:1004.1287v2. 
